\title{
ORIGINALS
}

\section{Reaktion von reduzierter A- und B-Kette mit Insulin [15]}

\author{
Helmut Zahr, Bernd Gutte* und Hans-Gregor Gattren**
}

Deutsches Wollforschungsinstitut an der Technischen Hochschule Aachen

\author{
Eingegangen am 27. Dezember 1967
}

\section{Reactions of reduced $A$ - and $B$-chains with Insulin}

Summary. When insulin is reacted with bis-thiol-Bchain (prepared from beef insulin) in a molar ratio $1: 1$ at pH 9.5 in the presence or absence of air, oligomeric and polymeric chains, linked by disulfide bonds, are formed. Equimolar amounts of insulin-A-chain in its tetrathiol form when brought into reaction with insulin at $\mathrm{pH} 9.3$ in the presence of air also cause a partial transformation of insulin into oligomeric and polymeric products. Partially oxidized A-chain, however, containing an average of 2 moles SH-groups per mole of A-chain, does not attack insulin under these reaction conditions.

Réaction, avec l'insuline, des chaînes $A$ et $B$ réduites

Résumé. Quand l'insuline réagit avec la chaîne $B$ bithiol (préparée à partir de l'insuline de boeuf) dans un rapport molaire $1: 1$, à $\mathrm{pH} 9.5$, en présence ou en l'absence d'oxygène de l'air, il se forme des chaînes oligomères et polymères reliées par des liaisons disulfure. - Des quantités équimolaires de chaîne-A d'insuline, sous sa forme tetrathiol, provoquent également une transformation partielle de l'insuline en produits oligomères et polymères, quand elles sont mises en réaction avec l'insuline à $\mathrm{pH}$
9.3, en présence d'oxygène de l'air. Cependant, la chaîneA partiellement oxydée, contenant en moyenne $\mathbf{2}$ moles de groupement $\mathrm{SH}$ par mole de chaîne A, n'attaque pas l'insuline dans ces conditions de réaction.

Zusammenfassung. Aus Rinderinsulin isolierte B-Kette in der Bisthiolform reagiert mit Insulin bei der Umsetzung im Molverhältnis 1:1 und pH 9.5 sowohl in Gegenwart als auch bei Ausschluß von Luftsauerstoff unter Bildung von oligomeren und polymeren über Disulfidbindungen verbundenen Ketten. - Auch Insulin-A-Kette in der Tetrathiolform wandelt Insulin bei $\mathrm{pH} 9.3$ in Gegenwart von Luftsauerstoff teilweise in oligomere und polymere Produkte um, wenn äquimolare Mengen in die Reaktion eingesetzt werden. Unter gleichen Reaktionsbedingungen greift partiell oxydierte A-Kette, die durchschnittlich 2 Mole SH-Gruppen pro Mol Kette enthält, Insulin nicht an.

Key-words: Beef insulin, reduced insulin-A-chain, partially oxidized insulin-A-chain, reduced insulin-Bchain, A-chain oligomers, B-chain polymers, thiol-disulphide interchange, thiol groups, reduction, stability, inactivation.
Ausgangspunkt der vorliegenden Arbeit war die Beobachtung [1, 21, 19], daß sich bei der oxydativen Resynthese von Insulin aus den reduzierten Ketten neben Insulin Polymere mit hohem B-Kettengehalt als Hauptprodukt bilden. Hatten wir zunächst angenommen, diese Polymere würden in einer Nebenreaktion durch Oxydation der einzelnen Ketten entstehen, so lernten wir bald, daß Kettenpolymere auch dann auftreten, wenn die B-Kette nicht als Thiol, sondern als S-Sulfonat [18] mit reduzierter A-Kette unter Luftausschluß kombiniert wird. Später wurde gefunden $[10$, 23], daß polymere Kettendisulfide sogar auftreten, wenn man Insulin mit 50 Äquivalenten Mercaptoäthanol behandelt. Die einfachste Erklärung dieser Befunde ist die Annahme von Grvol et al. [11], daß das unsymmetrische Disulfid Insulin ein instabiles Disulfidsystem darstellt, welches von Mercaptoverbindungen leicht angegriffen wird, wobei sich durch ThiolDisulfidaustauschreaktionen oligomere oder polymere Kettendisulfide bilden. Die Tendenz zur Bildung dieser stabilen Polymeren ist so groß, daß eine vollständige Reduktion von Insulin nur bei einem sehr großen Überschuß von Reduktionsmittel gelingt.

* Teil der Dissertation B. Gưre, Technische Hochschule Aachen 1967

** Teil der Dissertation H.-G. Gatrmer, Technische Hochschule Aachen 1967
Das Reduktionsmittel setzt primär Insulinthiolgruppen und Thiolketten frei, die als eigentliche Reaktanten der Polymerreaktion anzusehen sind.

Wenn diese Vorstellung richtig ist, sollten isolierte Ketten in der Thiolform mit Insulin dieselben Polymere liefern wie fremde Thiole, z. B. Mercaptoäthanol.

Im Zusammenhang mit unseren Insulin-Resyntheseversuchen [20] war es daher interessant zu prüfen, ob Insulinketten in der Thiolform bereits unter Resynthesebedingungen und bei einem Molverhältnis von $1: 1$ reagieren.

Bei der Behandlung von Insulin mit einem achtfachen molaren Überschuß an reduzierter A-Kette fanden Katsoyansis et al. [13] kürzlich ebenfalls einen Abbau des Insulins.

\section{Methoden und Ergebnisse}

1. Reaktion von Thiol-B-Kette mit Insulin. Man löste $50 \mathrm{mg}$ kristallisiertes Rinderinsulin (Hoechst) und $30 \mathrm{mg}$ frisch reduzierte B-Kette [12] in $16 \mathrm{ml} 0.05 \mathrm{M}$ Ammoniumhydrogencarbonatpuffer (pH. 9.5) und ließ den Ansatz bei $5^{\circ}$ stehen. Nach 24 Std waren keine SH-Gruppen mehr nachweisbar. Die Reaktionsmischung wurde eine Stunde gegen dest. Wasser dialysiert. Nach Gefriertrocknung resultierten $75 \mathrm{mg}$ pulverförmiges Präparat (94\% der eingesetzten Reaktanten). 
Das Lyophylisat lieferte bei der Papierelektrophorese im sauren Harnstoffpuffer nach Du et al. [5] drei Banden (vgl. Abb. 1).

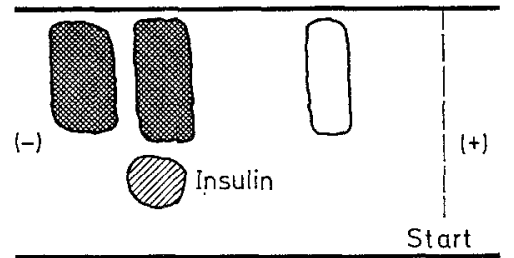

Abb. 1 Elektropherogramm der Reaktionsprodukte aus der äquimolaren Umsetzung von reduzierter B-Kette $\left(\mathrm{B}(\mathrm{SH})_{2} \text { ) mit Insulin (AB(SS) }\right)_{3}$ *. Elektrolyt: $2.4 \mathrm{M}$ Ameisensäure - $4 \mathrm{M}$ Harnstoff, $\mathrm{pH} 2.7,7$ Volt $/ \mathrm{cm} / 6$ Std., Entwicklung mit Pauly-Reagenz

Durch Gelfiltration an Sephadex G-50 fine wurden $40 \mathrm{mg}$ des Rohproduktes in drei Fraktionen aufgetrennt, die nach Dialyse und Gefriertrocknung zusammen $35.7 \mathrm{mg}$ Protein ergaben (vgl. Abb. 2).

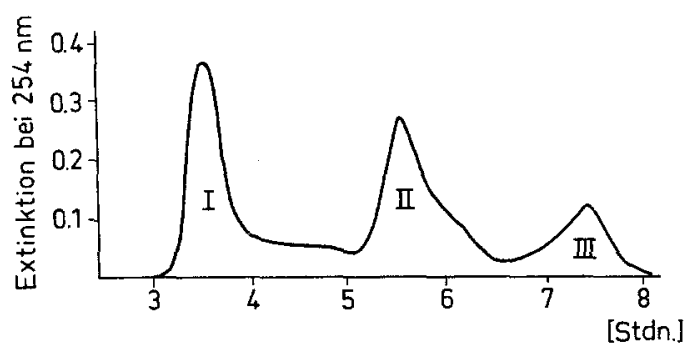

Abb. 2. Gelfiltration von $40 \mathrm{mg}$ der Reaktionsprodukte aus der äquimolaren Umsetzung von $\mathrm{B}(\mathrm{SH})_{2}$ mit $\mathrm{AB}(\mathrm{SS})_{3}$ an Sephadex G-50 fine. Säule: $100 \times 1.9 \mathrm{~cm}$. Elutionsmittel: $0,05 \mathrm{M}$ Ammoniumhydrogencarbonatpuffer, $\mathrm{pH}$ $8.2[7]$, Geschwindigkeit: $22 \mathrm{ml} / \mathrm{Std}$

Fraktion I enthielt polymere Produkte, die noch stärker kationisch geladen waren als Insulin. Die Oxydation mit Perameisensäure [17] und die Elektrophorese der oxydierten Produkte bei $\mathrm{pH} 2$ ergaben, daß sich diese überwiegend aus B-Kette und wenig A-Kette zusammensetzte.

Fraktion II bestand aus fast reinem Insulin, das in einer Ausbeute von $12.5 \mathrm{mg}$ zurückgewonnen wurde. Dies sind $35 \%$ der isolierten Proteine. Der Insulingehalt des Reaktionsproduktes vor der Fraktionierung betrug aufgrund des Testes am Rattenfettgewebe etwa 30\%. Dieser Wert stimmt mit dem Ergebnis der Fraktionierung gut überein.

Ausgegangen wurde von einem Gemisch aus Insulin und reduzierter B-Kette mit einem Insulingehalt von $62.5 \mathrm{Gew} \%$. Aufgrund der gravimetrisch ermittelten Abnahme des Insulingehaltes von 62.5 auf $35 \%$ wur-

* A und B stehen für die separaten Polypeptidketten des Insulins ohne die Schwefelatome der Cysteinreste; $\mathrm{B}(\mathrm{SH})_{2}$ ist also die Thiol-B-Kette, $\mathrm{B}\left(\mathrm{SSO}_{3}^{-}\right)_{2}$ deren bis -S-Sulfonat. den $44 \%$ des eingesetzten Insulins durch die Einwirkung der Thiol-B-Kette abgebaut.

Die dritte Fraktion der Sephadex-G-50-Trennung enthielt hauptsächlich monomere Bis-disulfid-A-Kette $\left(\mathrm{A}(\mathrm{SS})_{2}[3,22]\right.$ neben Spuren von Insulin. A(SS), wie das B-Kettenpolymer [1, 21, 19] wurden durch ihr Elutionsvolumen und ihr Verhalten bei der Elektrophorese aufgrund der bekannten Daten von authentischen Verbindungen [3, 22, 1, 21, 19] identifiziert.

Die oben beschriebene Umsetzung wurde bei einer Reaktionsdauer von 24 Std unter Resynthesebedingungen, d.h. in Gegenwart von Luftsauerstoff, ausgeführt. Um festzustellen, wie rasch Insulin durch reduzierte B-Kette abgebaut wird, ließ man $35 \mathrm{mg} \mathrm{B}(\mathrm{SH})_{2}$ mit $60 \mathrm{mg}$ Insulin (Molverhältnis 1:1) in $20 \mathrm{ml} 0.2 \mathrm{mo}-$ larem Ammoniumformiat/Ammoniak-Puffer vom $\mathrm{pH}$ 9.5 eine Stunde unter Luftausschluß reagieren. Das Reaktionsgemisch wurde mit halbkonzentrierter Ameisensäure auf $\mathrm{pH} 8$ eingestellt, und die Thiolgruppen wurden durch Zugabe von Jodessigsäure nach CRESTFIELD et al. [2] carboxymethyliert. Nach Dialyse und Gefriertrocknung erhielt man $76 \mathrm{mg}$ weißes Pulver (80\% der eingesetzten Reaktanten). Durch Gelfiltration an Sephadex G-50 fine unter gleichen Bedingungen wie vorher wurde dieses Präparat wieder in drei Fraktionen aufgetrennt, deren Zusammensetzung den Fraktionen des ersten Versuchs entsprach. Fraktion I $(29 \mathrm{mg}$ $=45 \mathrm{Gew} . \%$ ) enthielt Polymerprodukte, Fraktion II $(23 \mathrm{mg}=35$ Gew. \%) bestand weitgehend aus Insulin, und Fraktion III (13 mg $=20$ Gew. $\%)$ enthielt überwiegend A-Kettenderivate.

Fraktion III wurde durch Ionenaustauschchromatographie an Dowex $50 \times 2\left(\mathrm{Na}^{+}\right.$-Form) bei $\mathrm{pH} 4.5$ (Puffer: 0.2 M Ammoniumacetat/Essigsäure $+6 \mathrm{M}$ Harnstoff) von geringen Anteilen S-CarboxymethylB-Kette befreit. Die nach Dialyse und Gefriertrocknung des Eluats erhaltene Substanz enthielt nach der polarographischen Disulfidanalyse [16] $1.4 \mu$ Mole Cystin pro $\mu \mathrm{Mol}$ A-Kette. Die Papierelektrophorese bei $\mathrm{pH} 4.5$ ergab zwei Banden, die $\mathrm{A}(\mathrm{SS})\left(\mathrm{SCH}_{2} \mathrm{COOH}\right)_{2}$ $[10,23]$ und $\mathrm{A}(\mathrm{SS})_{2}[3,22]$ entsprachen.

Dieser Versuch zeigt, daß Insulin durch $\mathrm{B}(\mathrm{SH})_{2}$ weitgehend abgebaut wird. Aufschlußreich ist die Frage nach den durch die Reaktion von $\mathrm{B}(\mathrm{SH})_{2}$ mit Insulin freigesetzten Insulin-SH-Gruppen. Bei einer statistischen Reduktion wären alle denkbaren A-Kettenthiole bzw. ihre Carboxymethylderivate zu erwarten. $\mathrm{A}(\mathrm{SH})_{4}$ bzw. sein Carboxymethylderivat wurden aber nicht gefunden. Hauptprodukte sind vielmehr eine A-Kette mit einer Disulfidbindung und zwei Thiolgruppen sowie eine $A$-Kette mit zwei Disulfidbindungen.

2. Realtion von vollreduzierter A-Kette mit Insulin. $20 \mathrm{mg}$ aus Buntesalz-A-Kette gewonnene Thiolkette [12] wurden mit $50 \mathrm{mg}$ Insulin (Molverhältnis $\mathrm{A}(\mathrm{SH})_{4}$ : Insulin $=1: 1$ ) in $14 \mathrm{ml} 0.05$ molarem Glycinpuffer vom $\mathrm{pH} 9.3$ (Proteinkonzentration $5 \mathrm{mg} / \mathrm{ml}$ ) gelöst. Nach 28 Std bei $5^{\circ}$ waren SH-Gruppen mit der Nitroprussidreaktion nicht mehr nachweisbar. Der Ansatz 
wurde eine Stunde gegen dest. Wasser dialysiert. Nach Gefriertrocknung wurden $67 \mathrm{mg}$ Reaktionsprodukt (96\% der eingesetzten Reaktanten) erhalten, das nach der biologischen Prüfung (Blutzuckersenkung an der Ratte) $64 \%$ Insulin enthielt.

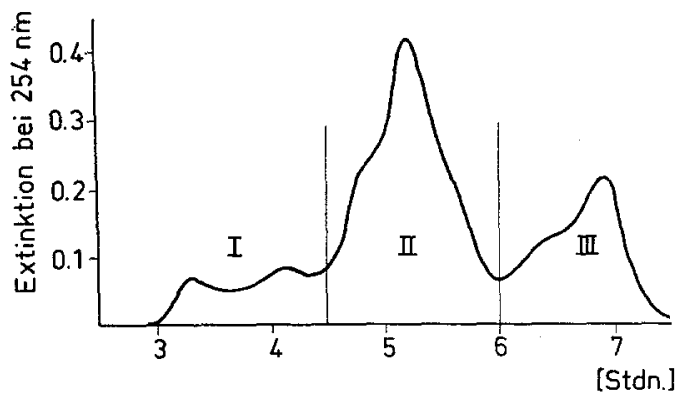

Abb. 3. Chromatographie von $65 \mathrm{mg}$ der Reaktionsprodukte aus der äquimolaren. Umsetzung von $\mathrm{A}(\mathrm{SH})_{4}$ mit Insulin an Sephadex G-50 fine. Säule: $100 \times 1.9 \mathrm{~cm}$. Elutionsmittel: 0,05 M NH $\mathrm{NCO}_{3}$-Puffer, $\mathrm{pH} 8.2 ; 23 \mathrm{ml} /$ Std

Die Auftrennung der Reaktionsprodukte in drei Fraktionen gelang durch Chromatographie an Sephadex G-50 fine (vgl. Abb. 3).

Die hochmolekulare Fraktion I bestand teilweise aus B-Kettenpolymeren, Fraktion II überwiegend aus Insulin und Fraktion III aus A-Kettendisulfiden.

Die quantitative Auswertung des Versuchs ergab folgendes: Das Gemisch der Reaktanten bestand $\mathrm{zu}$ $71 \%$ aus Insulin. Die Insulinfraktion (38 $\mathrm{mg}$ ) machte $61.5 \%$ der $62 \mathrm{mg}$ Protein aus, die insgesamt aus der Sephadexsäule eluiert wurden. Der Insulingehalt hat sich demnach infolge der Reaktion mit $\mathrm{A}(\mathrm{SH})_{4}$ von $71 \%$ auf $61.5 \%$ vermindert, dies sind etwa $14 \%$ Aktivitätsverlust. Dieser Wert stimmt mit dem Ergebnis der biologischen Prüfung gut überein.

Vollreduzierte A-Kette greift demnach Insulin langsamer an als reduzierte B-Kette. Als unwirksam erwies sich die präoxydierte A-Kette [20], die bei einer Umsetzung mit der äquimolekularen Menge Insulin bei pH 9.3 in 0.5-proz. Lösung nach 24 Std den Insulingehalt der Mischung nicht verringerte. Der Insulingehalt blieb unverändert $70 \%$.

\section{Diskussion}

Die experimentellen Ergebnisse der Reaktion zwischen reduzierter B- oder A-Kette und Insulin haben gezeigt, daß Insulin in Gegenwart seiner reduzierten Ketten bei pH 9 nicht stabil ist.

Sehr viele verschiedenartige Thiol-Disulfidaustauschreaktionen sind $\mathrm{z}$ wischen reduzierten Ketten und Insulin möglich. Z.B. kann aus reduzierter B-Kette (II) und Insulin (I) eine $\mathrm{AB}_{2}$-Verbindung (III) entstehen, die nach Angriff eines zweiten Moleküls reduzierter B-Kette eine $B_{3}$-Verbindung (IV) und monomere A-Kette mit der intakten 6.11-Disulfidbindung (V) liefert. Diese bildet bei Oxydation leicht ein cyclisches Dimeres $\mathrm{A}_{2}(\mathrm{SS})_{4}$ (VII), das $\mathrm{B}_{3}$-Produkt (IV) geht leicht durch Oxydation der freien SH-Gruppen in polymere, durch Disulfidbindungen verknüpfte B-Ketten (VI) über (Abb. 4).

Reagiert eine reduzierte A-Kette (VIII) mit Insulin, so können ein $\boldsymbol{A}_{2}$-Produkt (IX) und freie B-Kette (II) entstehen (Abb. 5).

Diese freie B-Kette greift intaktes Insulin wieder wie oben beschrieben an.

Bei der partiellen Mercaptolyse [10, 23] von Insulin werden reduzierte Ketten gebildet. Auch diese greifen intaktes Insulin auf gleiche Weise an, so daß schließlich ähnlich gebaute oligomere A-Ketten und polymere B-Ketten auftreten müssen.

Selbstverständlich kann es durch Oxydation von verschiedenen Zwischenprodukten zu Heteropolymeren aus B- und A-Ketten kommen, die wiederum durch Disulfidbindungen verknüpft sind.

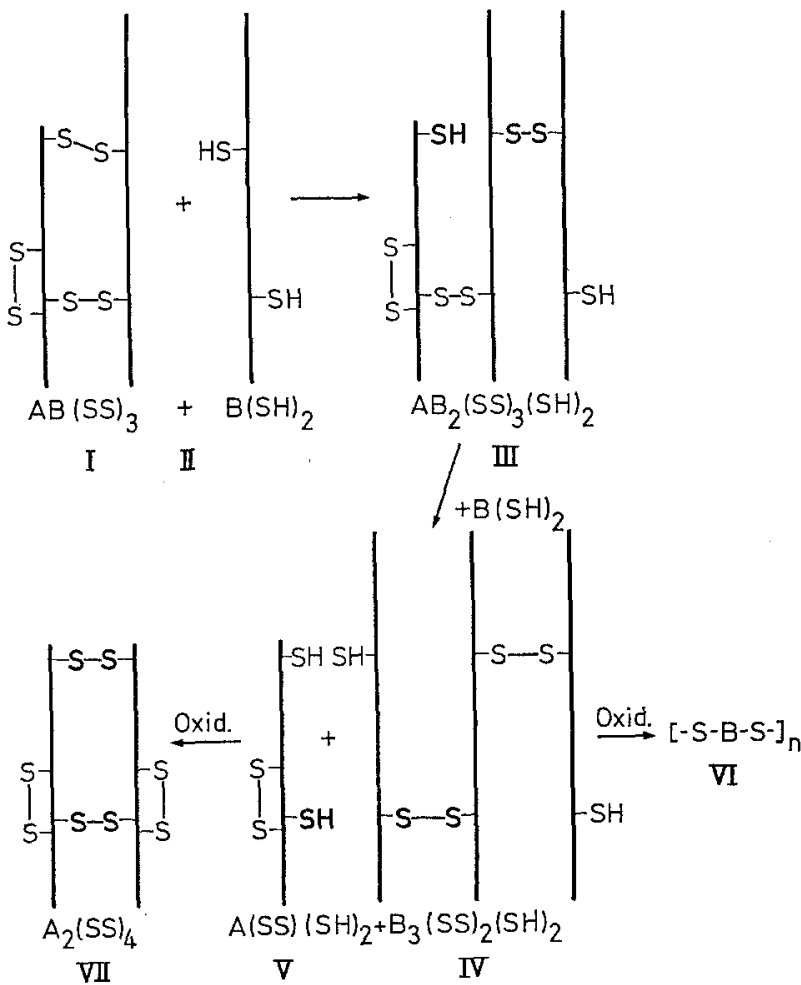

Abb. 4. Mögliche Reaktionen der reduzierten B-Kette mit Insulin

Die vorliegenden Versuchsergebnisse dürften eine Reihe von experimentellen Beobachtungen bei der Resynthese von Insulin aus seinen separaten Polypeptidketten erklären: Bei Anwendung der Footner-Smiles Reaktion [9] entstehen weniger polymere Ketten [15], weil die aggressive reduzierte B-Kette erst aus gemischten Disulfiden von A- und B-Kette durch Reaktion mit überschüssiger reduzierter A-Kette freigesetzt werden $m u ß$, die A-Kette aber weniger thiophil ist als B-Kette. Bei dem chinesischen A-Ketten-Überschußverfahren [4], das Katsoyannis und Tometsko [14] übernommen und modifiziert haben, trifft primär vor- 
handenes oder durch Disulfidaustausch freigesetztes $\mathrm{B}(\mathrm{SH})_{2}$ häufiger auf A-Kette als Reaktionspartner als auf Insulin.
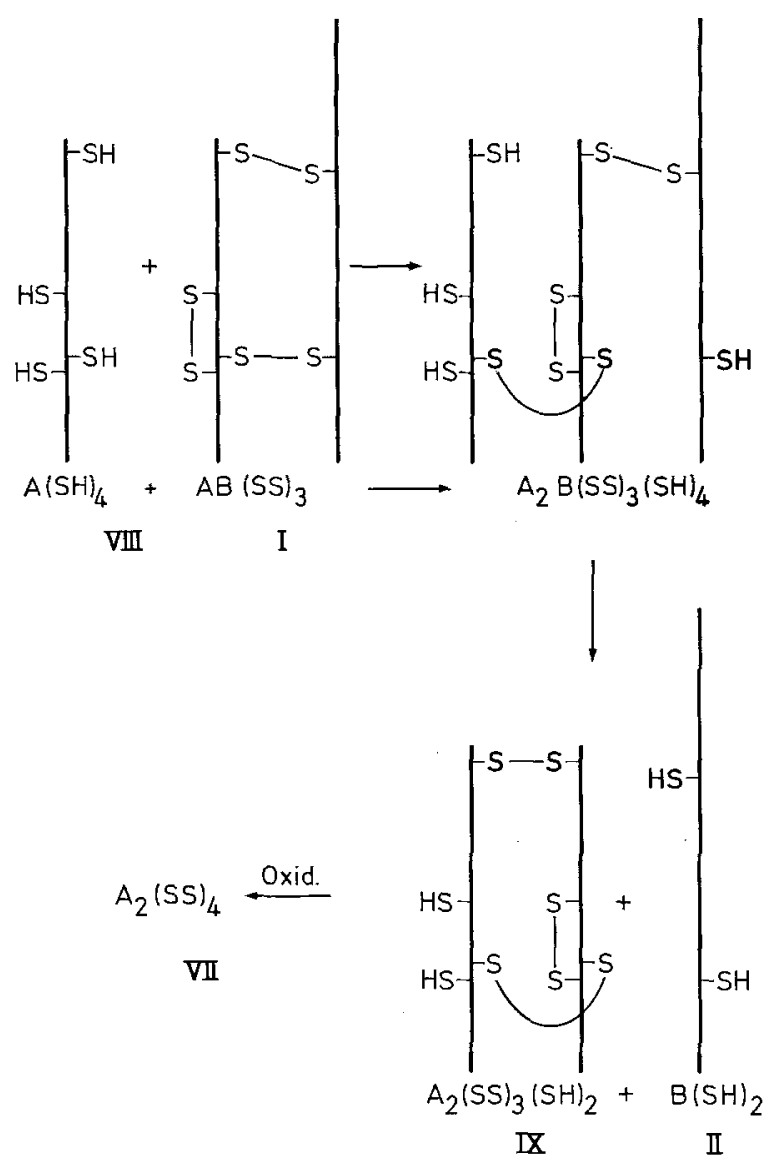

Abb. 5. Mögliche Reaktionen der reduzierten A-Kette mit Insulin

Der Erfolg der Präoxydationsmethode [20] beruht vermutlich auf der Tatsache, daß präoxydierte A-Kette gebildetes Insulin nicht angreift.

Darüber hinaus könnten unsere Ergebnisse auch einen Zusammenhang mit den Arbeiten von Ensivck et al. [6] und Feniches et al. [8] haben. Danach inhibiert ein Komplex aus reduzierter B-Kette mit kristallisiertem Serumalbumin die biologische Aktivität des Insulins. Fenichex et al. [8] erklären ihre Befunde durch eine direkte Wirkung der B-Kette auf Insulin. Eine direkte Wirkung reduzierter B-Kette in freier Form auf Insulin, nämlich Abbau des Hormons unter Bildung von oligomeren und polymeren Kettenprodukten, wurde in der vorliegenden Arbeit gezeigt.

Dank. Diese Arbeit wurde mit Mitteln des Bundesministers für wissenschaftliche Forschung (Forschungsvorhaben St.-N 213-66) gefördert.

Den Farbwerken Hoechst danken wir für die großzügige Spende von kristallisiertem Rinderinsulin. Herrn Professor Dr. E.F. Pfeiffer und Dr. J. Ammon sowie Herrn Dr. N. Puler danken wir sehr für die Durchführung der Insulinbestimmungen.

\section{Literatur}

1. BRINkноF', O.: Spaltung des Insulins in Glycyl- und Phenylalanylkette und Versuche zur Rekombination der Ketten. Diss. Techn. Hochschule Aachen 1964, zitiert bei ZahN, H., J. MeIEnhofer u. E. SChNABEL: Präparative und analytische Probleme bei der Aachener Insulinsynthese. Acta Chim. Acad. Sci. Hung. 44, $109-128(1965)$.

2. Crestrield, A.M., S. Moore, and W.H. Stern: The Preparation and Enzymatic Hydrolysis of Reduced and S-Carboxymethylated Proteins. J. biol. Chem. 238, 622-627 (1963).

3. Drechser, E.: Utber die partielle Sulfitolyse von Insulin und dessen Resynthese. Diss. Techn. Hochsch. Aachen 1967.

4. DU, Y.-C., R.-Q. JTang, and C.-L. Tsou: Conditions for successful Resynthesis of Insulin from its Glycyl and Phenylalanyl Chains. Scientia sinica 14, 229-236 (1965).

5. - Y.-s. Zhang, Z.-X. Lu, and C.-L. Tsou: Resynthesis of Insulin from its Glycyl and Phenylalanyl Chains. Scientia Sinica 10, 84-104 (1961).

6. Ensinck, J.-W., R.J. MaHLER, and J. VaLIANCEOwex: Antagonism of Insulin Action on Muscle by the Albumin-Bound B Chain of Insulin. Biochem. J. 94, 150-159 (1965).

7. Epstein, C.J., and C.B. ANminsen: The Use of Gel Filtration in the Isolation and Purification of Beef Insulin. Biochemistry 2, 461-464 (1963).

8. Fentchel, R.L., W.H. Bechmann, and H.E. ALBURN : Inhibition of Insulin Activity in Mitochondrial Systems and in Normal Rats by Reduced B ChainAlbumin Complex. Biochemistry 5, 461-466 (1966).

9. Footner, H.B., and S. Smtles: Reactions of Organic Thiosulfates. J. chem. Soc. (London) 127, 2887-2891 (1925).

10. Gattrer, H.-G.: Die partielle Reduktion des Insulins. Diss. Techn. Hochschule Aachen 1967.

11. Grvol, D., F. De Lorenzo, R.F. Goldberger, and C.B. Anfinsen: Disulfide Interchange and the ThreeDimensional Structure of Proteins. Proc. nat. Acad. Sci. (U.S.A.) 53, 676-684 (1965).

12. Gutre, B.: Kombination von natürlichen und synthetischen Insulinketten zu biologisch aktivem Insulin nach der Präoxydationsmethode und Cntersuchung der dabei auftretenden Nebenprodulate. Diss. Techn. Hochsch. Aachen 1967.

13. Katsoyannis, P.G., A.C. Trakatellis, St. Johnson, Clypde Zarut, and G. Schwartz: Studies on the Synthesis of Insulin from Natural and Synthetic A and B-chains. II. Isolation of Insulin from Recombination Mixtures of Natural A and B Chains. Biochemistry 6, $2642-2655(1967)$.

14. - , and A. Tomexsko: Insulin Synthesis by Recombination of A and B Chains: A highly efficient Method. Proc. nat. Acad. Sei. (USA) 55, 1554-1561 (1966).

15. Diese Befunde wurden auszugsweise bereits früher referiert, vgl. Klostermeyer, H., u. R.E. Humber: Chemie und Biochemie des Insulins. Angew. Chem. 78, $871-886$ (1966); Angew. Chem. Int. Ed. 5, 807-822 (1966).

16. LFACH, S.J.: Stoichiometry in the estimation of disulfide in intact proteins using mereuric chloride. Biochim. biophys. acta (Amsterdam) 33, 264-266 (1959).

17. SANGer, F.: Fractionation of Oxidized Insulin. Biochem. J. 44, 126-128 (1949).

18. ZAHN, H.: Struktur und Synthese von Insulin. Verhandlungen der Deutschen Gesellschaft für innore Medizin, S. 72. München: J. F. Bergmann 1967. Kongreß (1966), S. 800 . 
19. - Über Insulin. Arbeitsgemeinschaft für Forschung des Landes Nordrhein-Westfalen, Heft 161 (1966), S. 45 .

20. - B. Gutte, E.F. Pfelffer u. J. Ammon: Resynthese von Insulin aus präoxydierter A-Kette und reduzierter B-Kette. Justus Liebigs Ann. Chem. 691, $225-231$ (1966).

21. - - u. O. BRINkноF : Zur Chemie der Rekombination von Insulinketten zu biologisch aktivem Insulin. Angew. Chem. 77, 509 (1965).
22. -, u. E. Drechsex: Die partielle Sulfitolyse des Insulins. Hoppe Seylers Z. physiol. Chem., im Druck. 23. -, u. H.-G. GatTNER: Die partielle Reduktion des Insulins. Hoppe-Seylers Z. physiol. Chem., im Druck.

HeLMUT ZaHN

Deutsches Wollforschungsinstitut Technische Hochschule Aachen. 5100 Aachen 